DOI: $10.12737 /$ article_5967eb2c83b3f2.56254300

УДК 338.242

ИССЛЕДОВАНИЕ ВЛИЯНИЯ РИСКОВ НА УСТОЙЧИВОЕ ФУНКЦИОНИРОВАНИЕ РЕГИОНАЛЬНОГО ЛОГИСТИЧЕСКОГО ИНДУСТРИАЛЬНОГО ПАРКА

доктор экономических наук, профессор Е. И. Макаров ${ }^{1}$

кандидат экономических наук, доцент Ю. И. Ярославцева ${ }^{1}$

1 - Воронежский филиал ФГБОУ ВО «РЭУ им. Г.В. Плеханова», г. Воронеж, Российская Федерация

Издано при финансовой поддержке Российского гуманитарного научного фонда. Заявка № 16-02-00256

В условиях меняющихся геополитических условий на юго-востоке Европы и вызванных этим трансформаций грузопотоков между Европой и Азией актуализируется проблема создания региональной транспортно-логистической инфраструктуры, способной адекватно реагировать на современные реалии. Одним из трендов формирования такой инфраструктуры является строительство логистических индустриальных парков приграничных и транзитных регионов нашей страны,представляющих собой объединения элементов инфраструктуры с присущими признаками промышленных индустриальных парков. Эффективное функционирование подобных структур, будет зависеть не только от состава его резидентов, но и от того, насколько это формирование способно адекватно отвечать на динамично меняющиеся внешние условия. Поэтому, актуальной научной задачей является разработка научно-методического обеспечения их устойчивого функционирования. В рамках этой проблемы первостепенной является исследование рисков функционирования логистических индустриальных парков. В статье на основе генезиса категории «логистический индустриальный парк〉 исследуются факторы, влияющие на его устойчивое функционирование, анализируются риски функционирования логистического индустриального парка. Исследуются важнейшие характеристики рисков логистического индустриального парка, связанные с транзитной спецификой региона. Предлагается двухуровневая классификация рисков с делением на внешние и внугренние с акцентом на транзитную специфику региона и особенности грузопотоков, проходящих через экономическое пространство региона. Проведенный анализ специфики и выявленная классификация рисков, позволили сформулировать определение риска функционирования логистического индустриального парка транзитного региона как возможности возникновения неблагоприятных ситуаций в регионе, возникающих в результате воздействия факторов нестабильности, приводящих к неустойчивой работе и невыполнению своих функций по продвижению грузопотоков через транзитный регион. Для управления рисками предлагается авторская трактовка схемы алгоритма, позволяющая повысить устойчивость функционирования логистического индустриального парка.

Ключевые слова: региональная транспортно-логистическая инфраструктура, логистический индустриальный парк.

\title{
THE RESEARCH OF RISKS INFLUENCE ON THE STABLE FUNCTIONING OF REGIONAL LOGISTICS INDUSTRIAL PARK
}

DSc in Economics, Professor E. I. Makarov ${ }^{1}$

PhD in Economics, Associate Professor Yu. I. Yaroslavtseva ${ }^{1}$

1 - Voronezh branch of Federal State Budget Educational Institution of Higher Education «Plekhanov Russian University of Economics», Voronezh, Russian Federation

Published with the financial support of Russian Humanitarian Scientific Fund. Application No. 16-02-00256

\begin{abstract}
In the face of changing geopolitical conditions in the South-East Europe and the consequent transformation of cargo flows between Europe and Asia, the problem of creation of regional transport-logistic infrastructure, able to adequately respond to modern realities becomes urgent. One of the trends of formation of such infrastructure is the construction of logistics industrial parks in border and transit regions of our country, representing the joint of elements of infrastructure with the inherent features of
\end{abstract}




\section{Управление. Моделирование. Информатика}

industrial parks. The effective functioning of such structures will depend not only on the composition of its residents, but also on how this formation is able to respond adequately to rapidly changing external conditions. Therefore, the current research objective is to develop scientific and methodological support of their sustainable functioning. In relation to this issue the study of the risks of functioning of logistic industrial parks is the main task. The article, on the basis of the genesis of the category of logistics industrial park, explores the factors that influence its sustainability, analyzes the risks of functioning of logistic industrial park. The most important characteristics of the risks of logistic industrial park associated with the transit specific region are researched. We propose two-tier risk classification with the division into external and internal ones, with a focus on transit specifics of the region and characteristics of cargo flows passing through the economic space of the region. Analysis of specifics and identified classification of risks, has allowed formulating the definition of operational risk of logistic industrial park of transit region, as the possibility of adverse situations in the region, resulting from influence of factors of instability, leading to unstable operation, and failure of its functions for promotion of traffic through the transit region. Author's interpretation of the scheme of the algorithm for risk management, which allows increasing the stability of functioning of logistic industrial park, is suggested.

Keywords: regional transport-logistic infrastructure, logistic industrial park.

Ранее в своих работах мы исследовали новую зарождающуюся организационную форму логистической инфраструктуры - логистические индустриальные парки, вкладывая в это понятие нечто большее, чем просто логистический центр огромных размеров. Под логистическим индустриальным парком мы понимаем комплекс объектов логистической инфраструктуры, дополненный системой профильных производств и услуг, расположенных на специально обустроенной площадке, обеспеченной инженерными коммуникациями, и функционирующий в условиях правового поля государственной, административной и правовой поддержки [1].

Логистический индустриальный парк (ЛИП) принципиально отличается от логистических кластерных структур, исследованных в работаx $[2,3,4,5]$. ЛИП представляет собой компактное объединение элементов инфраструктуры, присущее всем видам промышленных или индустриальных парков и другим формам кооперации хозяйствующих субъектов в современных условиях. ЛИП, так же как и региональные хозяйствующие субъекты, функционирует в рамках существующего правового поля $[6,7]$.

Как показала практика, эффективное функционирование таких структур, будет зависеть не только от состава его резидентов, но и от того, насколько это формирование способно адекватно отвечать на динамично меняющиеся внешние условия, которыедостаточно подробно исследованы нами для кластерных образований в работах $[8,9,10]$.
Логистический индустриальный парк региона, находящегося в узле международных транспортных коридоров, является сложной многоуровневой системой, функционирующей в условиях динамично прогрессирующей рыночной конъюнктуры. В современном формате развития национальной экономики транспортнологистическая сфера и сама становится одной из наиболее динамичных отраслей хозяйственной деятельности. Ее роль трудно недооценить, поскольку она стимулирует развитие инвестиционной активности и улучшает деловой климат в регионе.

На устойчивое и надежное функционирование логистического индустриального парка транзитного региона существенно влияют риски [11].

На современном рынке транспортнологистических услуг, характерными признаками функционирования предприятий стали неопределенность и неоднозначность хозяйственных ситуаций. Источниками нестабильности, а следовательно и факторами устойчивого функционированиялогистического индустриального парка, как и любого хозяйствующего субъекта, служат изменения спросаи предложения на транспортнологистические услуги в конкретном регионе, а также технические и маркетинговые просчеты в оценке ассортимента и объемов материальных потоков, неточности в оценке статистических данных, непрофессиональныедействия менеджеров, искажения в передаче данных и их интерпретации, а в последнее время, к ним присоединились 
также изменения геополитических условий. Стабильное функционирование ЛИП во многом зависит от характера и состава потока товаров, протекающего по территории региона [1].

Очевидно, что в нынешних условиях инфраструктура рынка региона невозможна без становления и развития устойчивых и надежно функционирующих образований хозяйствующих субъектов различных организационно-правовых форм. Поэтому учет факторов нестабильности и риска в современных условиях хозяйствования такой структуры, как логистический индустриальный парк, является актуальной научной проблемой.

Следует отметить, что всовременной научной литературе отсутствует единое определение понятия риск, научное понимание его содержания. С одной стороны, риск - это объективносубъективная экономическая категория, которая отражает уровень просчета или успеха в достижении намеченной цели с учетом влияния внутренних (условно-контролируемых) и внешних (условно-неконтролируемых) факторов, а с другой стороны, риск - это мера неопределенности и конфликтности в хозяйственной деятельности.

Исследованию различных аспектов проблемы рисков в логистических системах посвящены работы $[6,7,9,11]$.

Функционированиелогистического индустриального парка транзитного региона сопряжено со многими видами рисков, которые обусловлены условиями формирования логистического индустриального парка и факторами, влияющими на цепи поставок, создающими виртуальнопотенциальную или реально существующую опасность разрушения порядка его деятельности. При этом одной из важнейших характерныхособенностей рисков ЛИП является их «транзитная» специфика. В связи с этим возникает необходимость классификации рисков с акцентом на указанную специфику и особенности грузопотоков, проходящих через экономическое пространство региона.

Проанализировав условия создания и функционирования логистического индустриального парка транзитного региона, можно выделить внешние и внутренние риски, которые влияют на стабильность егодеятельности. Внешние риски, в свою очередь, подразделяются на макро- и микро, или внутристрановые. Деятельность логистического парка подвержена влиянию многих факторов внешней и внутренней среды транзитного региона. Именно это, а также характер грузопотоков, обслуживаемых в регионе, требует выделения разных уровней классификации рисков.

Исследование специфики и выявленная иерархия рисков ЛИП позволили нам сформулировать, достаточно приближенно, определение категории риска функционирования логистического индустриального парка транзитного региона. Риск ЛИП - это некоторая расчетная вероятность возникновения неблагоприятных ситуаций во внешней и внутренней среде, возникающих, в свою очередь, в результате воздействия факторов нестабильности, приводящих к неустойчивой работе и невыполнению своих функций по продвижению грузопотоков через транзитный регион.

Для регионов большое значение имеют международные транспортные коридоры. Их функциями является обслуживание экспортноимпортных и транзитных грузопотоков. Риски функционирования логистического индустриального парка региона, находящегося на пересечении международных транспортных коридоров, заключаются в совместном воздействии групп рисков на каждый вид грузопотоков (внутрирегиональных, экспортно-импортных, транзитных).

На формирование транзитного и импортного грузопотоков, поступающих из других стран и регионов оказывают воздействие макро- (мировые) и микро- (внутристрановые) риски. Однако данные группы рисков воздействуют и на региональные процессы, возникающие в связи с необходимостью учета угроз при поступлении грузопотоков и последующим продвижением грузов в регионы назначения.

Воздействие региональных рисков также распространяется не только на внутрирегиональные, но и определяет функциональные возможности логистического парка по выполнению необходимых транспортно-логистических операций с внешними импортно-экспортными грузопотоками. Анализ научной литературы в области фор- 


\section{Управление. Моделирование. Информатика}

мирования кластерных образований, а также незначительный опыт создания ЛИП в России позволил констатировать наличие синергетического риска, воздействующего на грузопотоки и объекты транспортно-логистической инфраструктуры региона.

Существует большая проблема, связанная с поиском научных методов определения влияния синергетического эффекта риска на результаты функционирования и развития логистического индустриального парка транзитного региона. В этой связи необходимо подчеркнуть, что выявление последствий воздействия каждой группы рисков на логистические цепи является сложным процессом, который определен необходимостью анализа влияния различных рисков на каждое звено региональной цепи поставок в контексте определенных грузопотоков региона.

Современное состояние региональной логистической инфраструктуры требует поиска новых методов повышения их устойчивости и конкурентоспособности, что, в свою очередь, должны обеспечивать эффективные механизмы управления риском. Данный факт вызывает необходимость разработки алгоритма определения влияния групп рисков на выполнение логистических процессов в цепях поставок регионального ЛИП и последствий этого влияния, которые находятся в причинноследственной связи. Применение разработанного алгоритма позволит определить воздействие каждой группы рисков на логистические процессы, протекающие как внутри ЛИП, так и вне его.

При рассмотрении рисков ЛИП возникает необходимость учета рисков функционирования не только его в целом как элемента инфраструктуры, но и рисков каждого его участника, так как процесс управления - это воздействие с целью нивелирования различий между состояниями «то, что должно быть» и «то, что есть». Этот риск проявляется в различиях условий хозяйствования предприятия, до и после его вступления в состав ЛИП.

Рассмотренные нами выше риски ЛИП находят свое конкретное выражение в производственно-финансовых результатах деятельности каждого резидента ЛИП. Как известно, деятельность любого хозяйствующего субъекта характеризуется такими наиболее значимыми объемными показателями, как товарооборот, грузооборот, объемы перевозок, а также финансовыми показателями, такими как рентабельность, прибыль, себестоимости единицы продукции или оказываемых услуг. Таким образом, такие последствия финансовых рисков, как увеличение издержек на выполнение транспортно-логистических операций, увеличение сумм контрактов, ухудшение финансовых условий резидентов ЛИП, прямо влияют на финансовые показатели каждого его участника. В свою очередь, для всех других видов риска и их последствий имеют место аналогичные рассуждения.

Таким образом, возникает необходимость говорить о втором уровне рисков ЛИП, возникающих у каждого резидента индустриального парка. Они состоят в некоторой вероятности ухудшения показателей производственнофинансовой деятельности хозяйствующего субъекта в случае его вхождения в состав ЛИП.

Очевидно, что в конечном счете именно результаты деятельности каждого предприятияхозяйствующего субъекта определяют его позицию по отношению к участию в составе ЛИП. Предприятие будет участвовать, если производственно-финансовые условия в рамках ЛИП лучше, чем вне его. И наоборот, предприятию будет выгоднее работать вне парка, если производственнофинансовые условия в парке будут хуже.

Ранее нами был рассмотрен ряд факторов, воздействующих на функционирование логистического индустриального парка транзитного региона, обуславливающих большую вероятность возникновения в нем рисковых ситуаций. Поэтому разработка и применение эффективных методов управления рисками является основополагающей задачей в методологии создания и функционирования ЛИП.

По мнению ряда авторов, в общем виде управление рисками включает несколько основных этапов: определение рисков, оценка рисков, разработка мероприятий по снижению рисков, контроль рисков, финансирование мероприятий по снижению рисков [11]. Схема алгоритма управления рисками для условий логистического индуст- 
риального парка транзитного региона с учетом транзитной специфики и двух уровней рисков будет выглядеть, как показано на рис. 1.

Действия по уменьшению уровня риска можно разбить на два важнейших направления: снижение уровня каждого вида риска и уменьшение возможных негативных последствий риска. Для снижения уровня каждого вида риска необходимо проведение дополнительных маркетинговых, экономических и социологических исследований. Для уменьшения негативных последствий рисков применяются такие известные и апробированные в отечественной и мировой практике инструменты, как привлечение гарантов и соинвесторов, страхование.

По нашему мнению, приоритетным направлением развития логистики на уровне регионов является создание и развитие ЛИП, имеющих неоспоримые преимущества за счет использования компактного месторасположения основных логистических производств и услуг, удобных транспортных развязок, сложившейся региональной системы дистрибуции в розничной торговле, переориентации на рынки с недостаточным присутствием, реализации государственных программ по развитию промышленных парков.

Реализация подобных проектов будет зависеть от комплексного сочетания организационных, управленческих, правовых, финансовых и геополитических векторов их развития.

В свою очередь, сочетание проектов по созданию ЛИП с предложенными алгоритмами управления рисками будет являться условием их успешного функционирования.

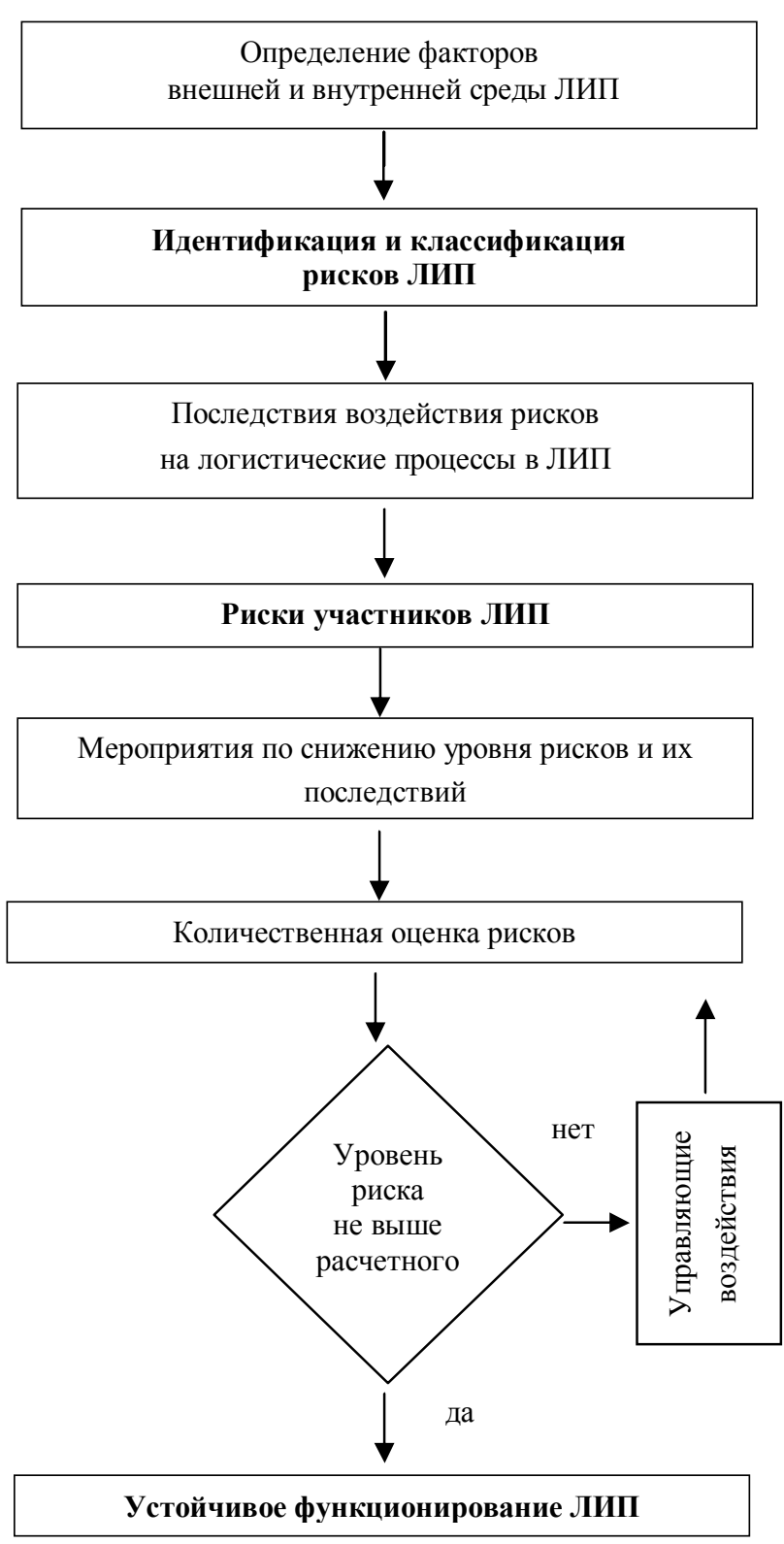

Рис. 1. Блок-схема алгоритма управления рисками логистического индустриального парка транзитного региона

\section{Библиографический список}

1. Макаров, Е. И. О некоторых аспектах проблемы создания регионального логистического индустриального парка [Текст] / Е. И. Макаров, В. В. Шалин // Логистика. - 2015. -№ 11 (108).- С. 22-25.

2. State stimulation of development of small entrepreneurship in developing countries [Text] / O. N. Kusakina, N. V. Bannikova, S. S. Morkovina, T. N. Litvinova // European Research Studies Journal. - 2016. - T. 19. - № 2S. P. 276-284.

3. Morkovina, S. S. Cluster approach to basis of forms of cooperation of the state and entrepreneurship in the forestry management of the sparsely wooded region [Text] / S. S. Morkovina // Life Science Journal. - 2014. - T. 11. № 10 s. - P. 423-427.

4. Development of methodological approaches to the efficiency analysis of territorial-industry cluster formation in the forest sector [Text] / S. S. Morkovina, E. G. Popkova, M. S. Santalova, A. V. Konstantinov //Asian Social 


\section{Управление. Моделирование. Информатика}

Science. - 2014. - T. 10. - № 23. - P. 81-93.

5. Methodological approach to the identification of predictive models of socio-economic processes for investment and innovative development of enterprises [Text] / T. L. Bezrukova, S. S. Morkovina, B. A. Bezrukov, E. G. Popkova // World Applied Sciences Journal. - 2013. - T. 26. - № 1. - P. 20-27.

6. Гамов, А.Н. Классификация условий формирования транспортно-логистических кластеров, находящихся на пересечении международных транспортных коридоров [Текст] / А.Н. Гамов // Ресурсы, Информация, Снабжение, Конкуренция. - 2014. - № 1. - С.89-93.

7. Гамов, А. Н. Организационно-экономические предпосылки формирования модели устойчивости транспортно-логистического кластера [Электронный ресурс] / А. Н. Гамов // Управление экономическими системами. - 2014. - № 2. - Режим доступа : http://www.uecs.ru/logistika/item/2801-2014-03-07-11-09-25.

8. Гамов, А.Н. Моделирование статической устойчивости транспортно-логистического кластера [Электронный ресурс] / А. Н. Гамов // Управление экономическими системами. - 2014. - № 3. - Режим доступа : http://www.uecs.ru/index.php?option=com_flexicontent\&view=items\&id=2827:2014-03-27-07-10-15.

9. Макаров, Е. И. Исследование факторов устойчивости транспортно-логистического кластера [Текст] / Е. И. Макаров, А. Н. Гамов // Логистика. - 2014. - № 2. - С. 30-32.

10. Морковина, С. С Управление обеспечением устойчивого развития предпринимательских структур сферы торговли и услуг [Текст] / С. С. Морковина, Е. А.Панявина, И. А. Аджамян // Социально-экономические явления и процессы. -2013 . - № 5 (51). - С. 137-143.

11. Морковина, С. С. Механизм обеспечения устойчивого развития малого предпринимательства в условиях возникновения кризисных ситуаций в экономике [Текст] / С. С.Морковина, Е. А. Панявина // Социально-экономические явления и процессы. - 2011. - № 12 (34). - С. 201-205.

12. Морковина, С. С. Развитие региональной инновационной инфраструктуры в контексте обеспечения экономической безопасности национального хозяйства [Текст] / С. С. Морковина, Ю. Н. Степанова, О. И. Васильев // ФЭС: Финансы. Экономика. Стратегия. - 2015. - № 2. - С. 51-57.

\section{References}

1. Makarov E.I., Shalin V.V. O nekotorykh aspektakh problemy sozdaniya regional'nogo logisticheskogo industrial'nogo parka [On some aspects of the problem of creating a regional logistics industrial park] Logistika [Logistics]. 2015, no. 11 (108), pp. 22-25. (In Russian).

2. Kusakina O.N., Bannikova N.V., Morkovina S.S., Litvinova T.N. Statestimulation of development of small entrepreneurship in developing countries. European Research Studies Journal, 2016, Vol. 19, no. 2S, pp. 276-284.

3. Morkovina S.S. Cluster approach to basis of forms of cooperation of the state and entrepreneurship in the forestry management of the sparsely wooded region. Life Science Journal, 2014, Vol. 11, no. 10s, pp. 423-427.

4. Morkovina S.S., Popkova E.G., Santalova M.S., Konstantinov A.V. Development of methodological approaches to the efficiency analysis of territorial-industry cluster formation in the forest sector. Asian Social Science, 2014, Vol. 10, no. 23, pp. 81-93.

5. Bezrukova T.L., Morkovina S.S., Bezrukov B.A., Popkova E.G. Methodological approach to the identification of predictive models of socio-economic processes for investment and innovative development of enterprises. World Applied Sciences Journal, 2013, Vol. 26, no. 1, pp. 20-27.

6. Gamov A. N. Klassifikatsiya usloviy formirovaniya transportno-logisticheskikh klasterov, nakhodyashchikhsya na peresechenii mezhdunarodnykh transportnykh koridorov [Classification of conditions for the formation of transport and logistics clusters located at the intersection of international transport corridors] Resursy, Informatsiya, Snabzhenie, Konkurentsiya [Resources, Information, Supply, Competition].2014, no. 1. (In Russian).

7. Gamov A. N. Organizatsionno-ekonomicheskie predposylki formirovaniya modeli ustoychivosti transportnologisticheskogo klastera [Organizational and economic prerequisites for the formation of the stability model of a transport-logistics cluster]Upravlenie ekonomicheskimi sistemami [Management of economic systems] 2014, no. 2. Available at: http://www.uecs.ru/logistika/item/2801-2014-03-07-11-09-25 (In Russian). 
8. Gamov A. N. Modelirovanie staticheskoy ustoychivosti transportno-logisticheskogo klastera [Modeling the Static Stability of a Transport-Logistics Cluster] Upravlenie ekonomicheskimi sistemami [Management of economic systems.2014, no. 3. Availableat: http://www.uecs.ru/ index.php?option=com_flexicontent\&view=items\&id=2827:201403-27-07-10-15. (In Russian).

9. Makarov E.I., Gamov A. N. Issledovanie faktorov ustoychivosti transportno-logisticheskogo klastera [Investigation of the sustainability factors of the transport and logistics cluster] Logistika [Logistics]. 2014, no. 2. (In Russian).

10. Morkovina S.S., Panyavina E.A., Adzhamyan I.A. Upravlenie obespecheniem ustoychivogo razvitiya predprinimatel'skikh struktur sfery torgovli i uslug [Management of sustainable development of business structures in the sphere of trade and services] Sotsial'no-ekonomicheskie yavleniya i protsessy [Socio-economic phenomena and processes].2013, no. 5 (51), pp. 137-143. (In Russian).

11. Morkovina S.S., Panyavina E.A. Mekhanizm obespecheniya ustoychivogo razvitiya malogo predprinimatel'stva $v$ usloviyakh vozniknoveniya krizisnykh situatsiy $v$ ekonomike [The mechanism of sustainable development of small business in the face of crisis situations in the economy] Sotsial'no-ekonomicheskie yavleniya i protsessy [Socioeconomic phenomena and processes].2011, no. 12 (34), pp. 201-205. (In Russian).

12. Morkovina S.S., Stepanova Yu.N., Vasil'ev O.I. Razvitie regional'noy innovatsionnoy infrastruktury $v$ kontekste obespecheniya ekonomicheskoy bezopasnosti natsional'nogo khozyaystva [Development of regional innovation infrastructure in the context of ensuring economic security of the national economy] FES: Finansy. Ekonomika. Strategiya [FES: Finance. Economy. Strategy]. 2015, no. 2, pp. 51-57. (In Russian).

\section{Сведения об авторах}

Макаров Евгений Иванович - заведующий кафедрой управления социально-экономическими системами и бизнес-процессами Воронежского филиала Российского экономического университета имени Г.В. Плеханова, доктор экономических наук, профессор, г. Воронеж, Российская Федерация; e-mail: ea_makarov@mail.ru.

Ярославцева Юлия Ивановна - доцент кафедры управления социально-экономическими системами и бизнес-процессами Воронежского филиала Российского экономического университета имени Г.В. Плеханова, кандидат экономических наук, доцент, г. Воронеж, Российская Федерация; e-mail: contact020781@mail.ru.

\section{Information about authors}

Makarov Evgeniy Ivanovich - Head of the Department of Management of Social and Economic Systems and Business Processes of the Voronezh branch of Federal State Budget Educational Institution of Higher Education «Plekhanov Russian University of Economics», DSc in Economics, Professor, Voronezh, Russian Federation; e-mail: ea_makarov@mail.ru.

Yaroslavtseva Yulia Ivanovna - Associate Professor Department of Management of Social and Economic Systems and Business Processes, Voronezh branch of Federal State Budget Educational Institution of Higher Education «Plekhanov Russian University of Economics», PhD in Economics, Associate Professor, Voronezh, Russian Federation; e-mail: contact020781@mail.ru. 